\title{
Evaluation of Maternal Dietary n-3 LCPUFA Supplementation as a Primary Strategy to Reduce Offspring Obesity: Lessons From the INFAT Trial and Implications for Future Research
}

\author{
Dorothy Marie Meyer ${ }^{1}$, Christina Brei ${ }^{1}$, Bernhard Lorenz Bader ${ }^{2,3}$ and Hans Hauner ${ }^{1,2,3 *}$ \\ ${ }^{1}$ Institute of Nutritional Medicine, School of Medicine, Technical University of Munich, Munich, Germany, ${ }^{2}$ ZIEL-Institute for \\ Food and Health, Freising, Germany, ${ }^{3}$ Else Kröner-Fresenius-Center for Nutritional Medicine, Technical University of Munich \\ School of Life Sciences Weihenstephan, Freising, Germany
}

Preclinical research suggests that early exposure to LCPUFAs is associated with offspring health outcomes, although evidence in humans is rather unclear. In 2006, we

OPEN ACCESS

Edited by:

Michele Barone,

University of Bari Aldo Moro, Italy

Reviewed by:

Tianan Alan Jiang,

McCormick and Company, Inc.,

United States

Alison Colquhoun,

University of São Paulo, Brazil

*Correspondence:

Hans Hauner

hans.hauner@tum.de

Specialty section:

This article was submitted to

Clinical Nutrition

a section of the journal

Frontiers in Nutrition

Received: 28 May 2020

Accepted: 04 August 2020

Published: 15 September 2020

Citation:

Meyer DM, Brei C, Bader BL and

Hauner H (2020) Evaluation of

Maternal Dietary n-3 LCPUFA

Supplementation as a Primary

Strategy to Reduce Offspring Obesity:

Lessons From the INFAT Trial and

Implications for Future Research.

Front. Nutr. 7:156.

doi: 10.3389/fnut.2020.00156 established the Impact of Nutritional Fatty acids during pregnancy and lactation on early human Adipose Tissue development (INFAT) study, a prospective randomized controlled intervention trial that examined whether decreasing the n-6/n-3 LCPUFA ratio during pregnancy and lactation influences offspring adipose tissue development in children up to 5 years. Our results indicate that maternal supplementation with n-3 LCPUFAs does not reduce offspring obesity risk, which is in line with recent publications. This perspective describes the challenges and lessons learned from our clinical trial. We discuss key findings and critically evaluate differences in study design, methodology, and analyses across similar intervention trials that may partly explain heterogeneous results. Summarizing evidence from human trials, we conclude that $n-3$ LCPUFA supplementation should not be recommended as a primordial strategy to prevent childhood obesity. Instead, it remains unknown whether n-3 LCPUFA supplementation could benefit high-risk subgroups and some vulnerable maternal/child populations. The perspectives offered herein are derived largely from insights gained from ours and similar n-3 LCPUFA intervention trials and help to provide direction for future research that examines the impact of maternal nutritional exposure on offspring health and disease outcomes.

Keywords: childhood obesity, n-3 LCPUFA supplementation, maternal diet intervention, fetal programming, early obesity prevention, breast milk

\section{INTRODUCTION}

It is now well-established that perinatal exposure to environmental conditions can influence longterm health. This concept, known as fetal programming, has its origins in epidemiological studies performed by Dr. David Barker (1) and was further developed into the framework known as the "Developmental Origins of Health and Disease" (DOHaD) (2). The theory maintains that exposure 
to adverse environmental stimuli during critical developmental periods can permanently alter regulatory functions and metabolic homeostasis in early life, increasing the risk of chronic diseases later on (3). "Adverse stimuli" also include exposure to a nutrient-excess environment in utero. Observational studies explored whether maternal obesity and gestational weight gain influenced offspring health outcomes. Research demonstrated that children born to mothers with obesity were at higher risk for obesity themselves early in life $(4,5)$, with some showing an elevated risk over their entire life-course (6). The DOHaD hypothesis also gave rise to intervention studies that focused on primordial prevention of obesity by optimizing the maternal diet during pregnancy and lactation (7).

Changes in dietary patterns have led to a shift in the dietary ratio of $n-6 / n-3$ LCPUFA intake in industrialized countries worldwide, favoring foods rich in n-6 LCPUFA sources. Evaluating the effects of changes in fatty acid intake in pregnant women is particularly important, as experimental studies indicate that fetal exposure to dietary LCPUFAs can modulate metabolic pathways involved in gene expression, cell differentiation, and epigenetic modifications (8). Other experimental work provided evidence that arachidonic acid (AA, 20:4n-6) enhances fat cell differentiation at the preadipocyte stage $(8,9)$, mediated by AA-derived prostacyclin $(9,10)$. In contrast, eicosapentaenoic acid (EPA, 20:5n-3) and docosahexaenoic acid (DHA, 22:6n-3) confer inhibitory action on fat cell differentiation in a highly coordinated and active process through several pathways (11). Consequently, it was suggested that modifying the composition of dietary fatty acids in pregnancy and lactation could reduce obesity risk in offspring via a programming effect (12). This assumption was mainly based on studies in rodents (13), whereas evidence from human data was largely indirect (14).

Examining the relationship between early exposure to LCPUFAs and childhood obesity gave rise to the "proof of concept" study Impact of Nutritional Fatty acids during pregnancy and lactation on early human Adipose Tissue development (INFAT). From 2006 to 2014, we conducted the first human intervention trial that examined whether reducing the maternal n-6/n-3 LCPUFA ratio during pregnancy and lactation influences offspring adipose tissue development and therefore could be used as an early obesity prevention strategy (15-17). We achieved this by supplementing women with $1.2 \mathrm{~g}$ of $\mathrm{n}-3$ LCPUFAs (180 mg EPA and 1,020 mg DHA as well as $9 \mathrm{mg}$ vitamin E as an antioxidant) daily and reducing their dietary AA intake to the recommended range of $50-90 \mathrm{mg}$ per day. This report presents a review of key findings. We present INFAT in the context of similar intervention trials and critically evaluate differences in design and methodology that could account for inconsistent findings. Synthesizing insights gained from our study and others, we reassess whether evidence has borne out that early exposure to LCPUFAs influences obesity risk in childhood. Finally, we discuss several promising n-3 LCPUFA intervention strategies that focus on sub-groups and high-risk populations that could improve health outcomes for both mother and child.

\section{LESSONS LEARNED FROM THE INFAT STUDY: IMPLICATIONS FOR PRACTICE}

The INFAT study was designed as an open-label, randomized, controlled longitudinal intervention trial, approved by the Technical University of Munich Ethics Committee and is registered in ClinicalTrials.gov: ID NCT00362089.

A total of 208 women were recruited to participate in the study and randomized to a control $(n=104)$ or intervention $(n=$ 104 ) group. The primary endpoint was the sum of four skinfold thickness (SFT) measurements in infants, assessed four times in the 1st year of life $(15,16)$. A planned follow-up trial continued annually in offspring from 2 to 5 years old (17). An overview of the study, including the design and data collection at each timepoint from recruitment through 5 years, can be seen in Figure 1. A more detailed description of the study design and methods can be found in the respective publications.

Table 1 presents a description of the main findings of the primary and secondary outcomes, listed by topic. In this manuscript, we will focus primarily on the objectives of the initial and follow-up studies, which are the key findings in the first two publications in Table 1 under the heading Body Composition in Newborns, Infants, and Children. However, we also made use of our extensive data set by pooling the intervention and control groups to explore relationships between perinatal dietary macronutrient and fatty acid exposure and offspring body composition (18-20, 23). These results are summarized in Table 1.

A secondary interest was to identify biomarkers that could predict adiposity outcomes. To this end, we pooled our cohorts to investigate several metabolic and hormonal factors in maternal blood, cord blood, and breast milk, including adiponectin, leptin, and indices of insulin resistance (26-32). A description of findings from all secondary analyses is found in Table $\mathbf{1}$ (15-17).

The long-term follow-up afforded us the opportunity to observe that several relationships between perinatal determinants and offspring body composition change over time. For example, while significant associations between n-3 and n-6 LCPUFAs, leptin, and adiponectin in maternal blood, cord blood, and breast milk were present early in life $(19,28,30)$, they did not persist up to 5 years $(20,29,31,32)$, as summarized in Table 1. This is an important observation to consider in planning future longitudinal studies, as around 5 years is known as the age of adiposity rebound and is thought to be a critical age that can predict later obesity (33). Recent findings from a German population study confirmed this link by reporting that accelerated weight gain in the preschool years is predictive of overweight and obesity in adolescence (34).

Most importantly, our results did not indicate that reducing the ratio of n-6/n-3 LCPUFAs during pregnancy and lactation influences adipose tissue development in early childhood, arguing against its use as an effective strategy to reduce obesity risk (15-17). We also found no evidence that supports maternal n-3 LCPUFA supplementation to improve offspring cognitive outcomes (24) (Table 1). 


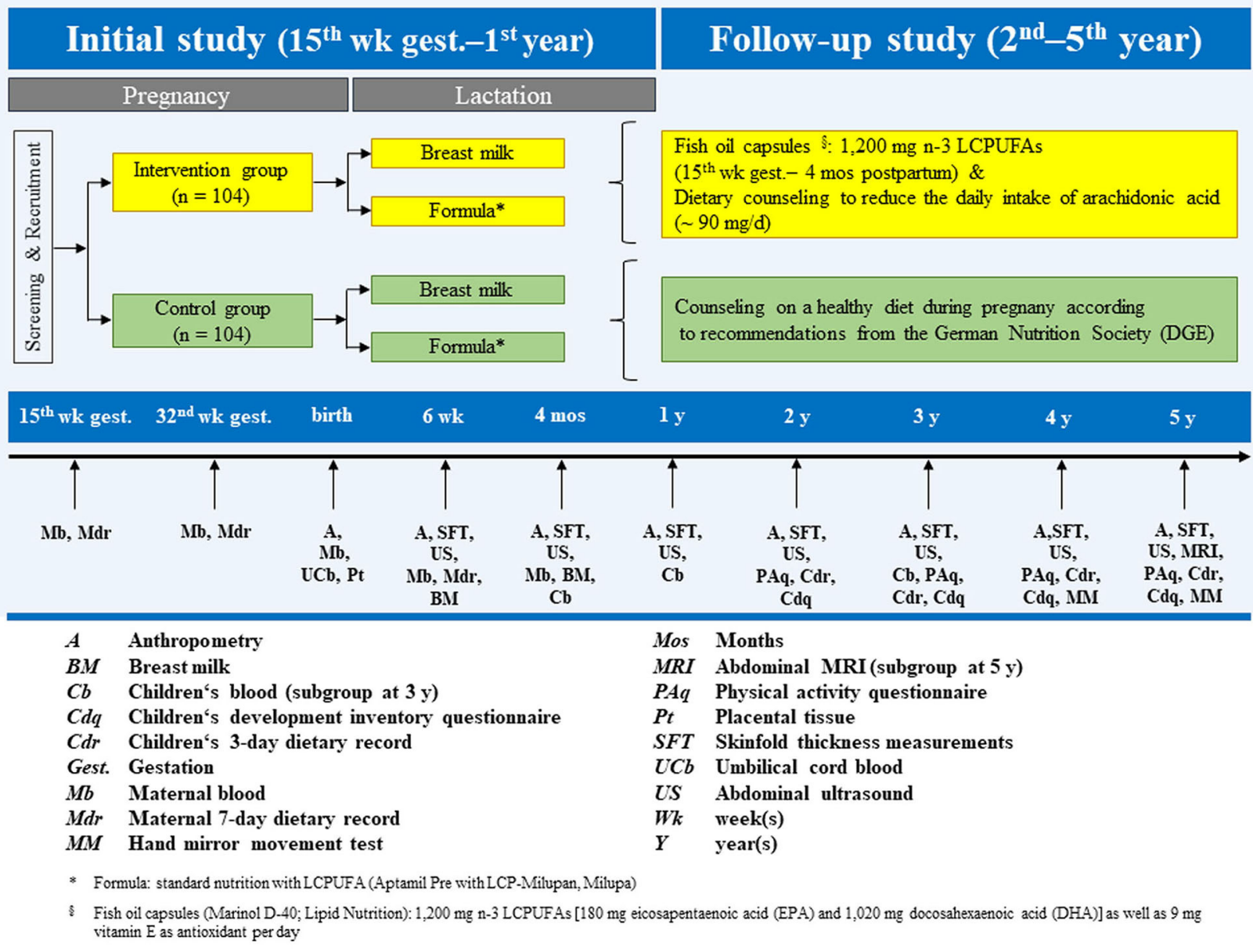

FIGURE 1 | INFAT study design, timeline, and overview of measurements and variables.

Many randomized controlled trials have assessed early childhood growth indirectly, using height and weight (35), BMI $z$-scores (36), and waist circumference (36) to estimate body composition. INFAT comprises one of the largest data sets of body composition and fat distribution measurements in children from birth to 5 years. An important contribution to the research community from INFAT was our assessment of body fat patterning and accretion via abdominal ultrasound throughout early childhood. We were the first to demonstrate that abdominal ultrasound was a reliable and reproducible method in preschoolers for measuring subcutaneous and preperitoneal fat $(21,22)$, the latter considered an approximation of visceral fat in children (37). Major advantages of ultrasound over other imaging techniques, such as MRI and dual-energy X-ray absorptiometry, are its portability and lower cost-per-examination, which make ultrasound a practical tool for measuring body fat in the clinical setting.

Estimating fat depots by abdominal ultrasound revealed that subcutaneous and preperitoneal fat develop at different rates and volumes in boys and girls $(21,22)$. INFAT girls had significantly larger measurements of subcutaneous fat than boys from birth up to 5 years, while differences in preperitoneal fat were first detectable from 3 years onward, with girls showing larger areas up to age 5. Concerning sex-specific effects and the importance of the placenta for fetal programming and growth, we revealed sexually dimorphic gene expression and n-3 LCPUFA responsiveness, with more pronounced effects in female placentas, especially in relation to the cell cycle (25). Analyzed placental gene expression correlated with birth weight but not with offspring adipose tissue growth in the first year (see description in Table 1). Our results underscore the importance of performing analyses to detect sex-specific differences and gain new insights into underlying mechanisms and sex-specific offspring growth.

\section{CRITICALLY APPRAISING THE INFAT RESEARCH DESIGN AND METHODOLOGY: CHALLENGES AND RECOMMENDATIONS FOR FUTURE STUDIES}

We assessed adherence to the fish oil capsule regimen and reduction of meat products with capsule protocol diaries and 7-day dietary records, respectively. Compliance was further 
TABLE 1 | Summary of INFAT publications by topic

Main outcome measures Study type

\section{Body Composition in Newborns, Infants, and Children}

A, SFT, US

Intervention vs. control

group

Birth, 6 wk, 4 mos, and $1 \mathrm{y}$

Maternal 7-day dietary records $s^{a, b}$ and maternal blood fatty acid profile ${ }^{a, b}$

Good maternal compliance of fish oil supplementation with the intervention group (assessed by capsule protocol, dietary records, and red blood cell FA analysis). Prolonged gestational duration in the intervention group by $\sim 5$ days. No evidence that $n-3$ LCPUFA supplementation in pregnancy and lactation affects offspring adipose tissue development or distribution.

Children's physical activity

No evidence that a dietary reduction of the maternal $n-6 / n-3$

questionnaires and dietary records $\mathrm{f}_{\mathrm{f}, \mathrm{h}}$ LCPUFA ratio has long-term effects on body composition in preschool children from 2 to 5 years. Influencing factors (physical activity, diet) could be excluded as confounding factors.

A, SFT, US Intervention vs. control

Birth, 6 wk, 4 mos, and $1 \mathrm{y}$ Fatty acid profile in maternal blood ${ }^{\mathrm{a}, \mathrm{b}}$ group and pooled cohort and $\mathrm{UCB}^{\mathrm{C}}$

Maternal n-3 LCPUFAs and n-6 LCPUFAs at 32 weeks' gestation were associated with birth weight and birth length. N-6 LCPUFAs were inversely related to $\mathrm{BMI}$ and ponderal index, but not fat mass, in the first year of life.

A, SFT, US Intervention vs. control group and pooled cohort

Birth, 6 wk, 4 mos, and 1 y $\quad$ Breast milk fatty acid profile ${ }^{\text {d,e }}$

The intervention was reflected in the breast milk FA profile; a positive relationship between the FA composition of breast milk and maternal blood was found. Early breast milk was related to the sum of four SFTs at 1 year.

A, SFT, US, MRI Pooled cohort 2, 3, 4, and $5 \mathrm{y}$

Adipose tissue measurement methods

Maternala ${ }^{a, b}, \mathrm{UCB}^{\mathrm{c}}$, and breast milk ${ }^{\mathrm{d}, \mathrm{e}}$ fatty acid profile

LCPUFAs in maternal plasma and UCB were not related to child outcomes. Increased breast milk n-3 LCPUFAs predicted several body composition measurements at 2 and 4 years. Breast milk $\mathrm{n}-6 / \mathrm{n}-3$ fatty acid ratio was inversely related to lean body mass at 4 and 5 years. Overall results do not provide sufficient evidence that LCPUFAs in maternal blood, UCB, and breast milk predict offspring adiposity up to 5 years.

Ultrasound was confirmed as a reliable method to estimate fat development and distribution in infants. Subcutaneous and preperitoneal abdominal fat grew at different rates, with an increase in preperitoneal fat in the first year, whereas subcutaneous fat areas increased from 6 weeks to 4 months, and decreased at 1 year postpartum. Sex-specific differences were observed for girls, with greater subcutaneous fat areas from 6 weeks onwards.

Adipose tissue measurement methods

Preperitoneal fat steadily increased in boys and girls over childhood, with larger volumes noted in girls from 3 years onwards. Subcutaneous fat decreased in both sexes at 2 years, then slightly increased from 2 to 5 years, with significantly larger volumes measured in girls at any timepoint investigated.

Dietary macronutrient intake during early gestation had no impact on offspring body composition. Maternal dietary fat at 32 weeks was inversely related to body composition at birth and 5 years. Higher carbohydrate and sugar intake in late pregnancy were associated with adiposity outcomes at 1 and 3 years. 
TABLE 1 | Continued

\section{Main outcome measures Study type \\ Neurological Development in Children}

Age at analysis

Main predictors and methods

Key findings

Reference

$\begin{array}{ll}\text { Neurological tests } & \text { Intervention vs. control } 4 \text { and } 5 \text { y } \\ & \text { group }\end{array}$

UCB LCPUFAs' ${ }^{c}$, child development inventory $\mathrm{g}^{\mathrm{gh}}$, hand mirror movement test ${ }^{g, h}$

\section{Placental Gene Expression}

A, SFT, US

Intervention vs. control

group (subpopulation

analysis) and pooled

subpopulations

\section{Maternal and Cord Blood Biomarkers}

A, SFT, US

Pooled cohort

A, SFT, US, MRI

Pooled cohort

Intervention vs. control group and pooled cohort

Birth, 6 wk, 4 mos, 1 and 2 Maternal insulin, triglycerides, and HOMA-IR ${ }^{1,2}$. UCB insulinc

\section{3,4 , and $5 y$}

Maternal insulin, triglycerides, and HOMA-IRa,b, UCB insulin ${ }^{\mathrm{C}}$

Birth, 6 wk, 4 mos, 1 and 2

Leptin, free leptin index, and soluble leptin receptor in maternal blooda,b,d, and $\mathrm{UCB}^{\mathrm{C}}$

Leptin in maternal blood ${ }^{\mathrm{b}}$ and $\mathrm{UCB}^{\mathrm{C}}$

6 wk, 4 mos, 1 and 2

3,4 , and $5 y$

Birth, 1, 2, 3, 4, and 5 y
Leptin and adiponectin in maternal blood ${ }^{\mathrm{d}, \mathrm{e}}$ and breast milk ${ }^{\mathrm{d}, \mathrm{e}}$

Leptin and adiponectin in breast milk ${ }^{\mathrm{d}, \mathrm{e}}$

HMW and total adiponectin in $\mathrm{UCB}^{\mathrm{C}}$ and child plasma
$\mathrm{N}-3$ LCPUFA supplementation in pregnancy and lactation is largely unrelated to neurological development at 4 and 5 years. Some weak associations were observed between UCB fatty acids and improved cognitive outcomes.

N-3 LCPUFA intervention in pregnancy has a more pronounced effect on female gene expression, especially in relation to the cell cycle and its associated modulator pathways. Significant changes in mRNA expression for CDK6, PCNA, and TGFN1 were observed in intervention group placentas. CDK6 and PCNA mRNA levels correlated with offspring birth weight and birth weight percentiles.

$\mathrm{N}-3$ LCPUFA responsive changes in placental gene expression do not influence offspring body composition.

Maternal metabolic markers were transiently related to child outcomes, but relationships were not consistently significant. Cord blood insulin was inversely related to weight gain up to 2 years, and this relationship was significant and stronger in girls only.

Maternal markers of glucose and lipid metabolism were unrelated to child outcomes. Lower cord blood insulin resulted in higher weight gain in girls at 5 years, but with very small effect sizes.

No effect of the intervention on maternal or cord blood leptin axis. Pooled analysis showed that maternal leptin at 32 weeks' gestation was inversely related to several body composition and growth outcomes at 2 years, while cord blood leptin was positively associated with child outcomes at birth and 2 years. Greater impact was detected for cord blood leptin.

Maternal leptin was inversely associated with growth in early childhood. Cord blood leptin was inversely and transiently related to certain growth and adipose tissue development indices in young children. Effect sizes were very small.

Breast milk adiponectin was associated with increased fat mass and weight gain up to 2 years.

Breast milk leptin and adiponectin were largely unrelated to body composition in children up to 5 years.

Neither HMW nor total adiponectin in cord blood were found to predict body composition in early childhood up to 5 years. No associations between $\mathrm{HMW}$ or total adiponectin, measured in child plasma at 3 years, and child growth or fat development at 3 , 4 , or 5 years were observed.
(24)

${ }^{\S}$ Publications with primary outcome analyses ${ }^{a} 15$ weeks' gestation, ${ }^{b} 32$ weeks' gestation, ${ }^{c}$ delivery, ${ }^{d} 6$ weeks postpartum, ${ }^{e} 4$ months postpartum, ${ }^{f} 3$ years, ${ }^{9} 4$ years, ${ }^{h} 5$ years.

Pooled cohort analyses: no evidence of a difference in analyzed parameters between intervention and control group was found. Hence, the two groups were pooled for further analysis.

A, Anthropometrics (e. g., body weight, body length or body height, BMI (body mass index) percentiles, head circumference, arm circumference, waist circumference); UCB, umbilical cord blood; HMW, high molecular weight; HOMA-IR, homeostatic model assessment of insulin resistance; FA, fatty acids; LCPUFA, long chain polyunsaturated fatty acids; mos, months; MRI, subcutaneous and visceral (cm ${ }^{3}$ ) adipose tissue development by magnetic resonance imaging (subgroup) at 5 years; pp, postpartum; RT-qPCR, quantitative reverse transcription polymerase chain reaction; SFT, adipose tissue development by sum of four skinfold thicknesses; US, subcutaneous and preperitoneal ( $m m^{2}$ ) adipose tissue development by ultrasound from 6 wk pp onwards; wk, weeks; y, years. 
confirmed by measuring the maternal fatty acid profile in blood and breast milk at four-time points during the study. Significant differences in LCPUFA levels between the intervention and control groups were observed in all maternal and infant tissues measured $(18,19)$, including plasma phospholipids, erythrocyte membranes, placenta, umbilical cord blood, and breast milk. There was also a reduced intake of AA from meat products and other foods in the intervention group at 32 weeks' gestation compared to the control group $(129.8 \pm 76.6$ vs. $160.6 \pm 80.6 \mathrm{mg}$ AA/d, $p<0.001$ ) (16). Overall, the n-6/n-3 LCPUFA ratio in the maternal diet was around 3.5:1 in the intervention and 7:1 in the control group (16), which was the ratio we hoped to achieve in the intervention group. Nevertheless, we were unable to confirm that achieving this ratio in pregnancy and lactation affects the accretion or distribution of adipose tissue in young children $(16,17)$. Our results are in line with two systematic reviews and a recent meta-analysis (38-40). Importantly, studies included in these analyses were very heterogeneous, both in investigative methods and primary outcomes, which raises questions as to whether differences in study design and analytical strategies may partly explain the lack of effects.

To our knowledge, we are the only research group whose primary aim was to balance the $n-6 / n-3$ LCPUFA ratio by supplementing n-3 LCPUFAs while concomitantly decreasing the maternal dietary intake of n-6 LCPUFAs. Most interventions have focused solely on increasing exposure to perinatal n3 LCPUFAs (40), which is worth discussing since a high background n-6 LCPUFA status limits the incorporation of n-3 LCPUFAs in phospholipids (41). Moreover, it is known that n-6 fatty acids can have a pro-adipogenic effect (9) and research indicates that maternal n-6 LCPUFA levels are strongly associated with offspring adiposity outcomes (42). This evidence suggests that studies with larger cohorts are needed to further explore whether clinically meaningful effects can be detected in offspring after reduced exposure to n-6 LCPUFAs in utero.

An important shortcoming of INFAT is that it was not adequately powered at the 5-year follow-up, which weakened our overall findings. The power calculation of $80 \%$ was based on a sample size that accounted for a drop-out rate of $30 \%$ at 12 months postpartum. We received additional funding during the first year to continue the follow-up period to 5 years postpartum. Notably, the total number of children for whom we had SFT measurements at 5 years was 114 , or $55 \%$ of the original sample. The sizeable attrition rate between 1 and 5 years can be partially explained because several families chose to drop-out after the initial study, despite the considerable effort made by INFAT researchers to encourage continued participation. Loss to followup is common in longitudinal studies, and ours was no exception. A pediatric obesity prevention study investigated reasons for attrition and found that overweight/obesity and related factors were the most salient determinants (43), a relevant finding for INFAT given that our primary outcome was adipose tissue mass measurements. Our experience with high drop-out rates emphasizes the need to clearly communicate intervention aims and follow-up duration with study participants at the onset of longitudinal studies.
Notably, when comparing perinatal intervention studies with similar aims, there are considerable differences in the dosage and composition of LCPUFAs, with little consensus on the optimal intake amount. The European Food and Safety Authority (EFSA) recommends a daily intake of $250 \mathrm{mg}$ DHA + EPA for adults, with an additional 100-200 mg of DHA during pregnancy and lactation (44). Dosage in intervention studies has ranged from $800 \mathrm{mg}$ to $4 \mathrm{~g}$ of $\mathrm{n}-3$ LCPUFAs per day and is mainly composed of DHA and EPA, usually with higher proportions of DHA $(40,45)$. We were concerned about the potential adverse effects of higher n-3 LCPUFA doses and selected $1.2 \mathrm{~g}$ of n-3 LCPUFAs (180 mg EPA and 1,020 mg DHA) as a compromise between efficacy and safety. Given the high variability in dosage and general lack of effects, it is possible that the dose of total LCPUFAs or the specific n3 LCPUFA composition was not high enough to observe the intended effect. Since designing our study, preclinical research has explored how different proportions of EPA and DHA in n3 supplements have varying effects on inflammatory pathways. Evidence shows that inflammatory markers are decreased at EPA/DHA ratios of $1: 1,1: 2$, and $2: 1(46,47)$, with the most pronounced effects observed at the ratio of 2:1 (47). Dasilva et al. (46) observed that EPA had increased antioxidant and anti-inflammatory capacities compared to DHA, and that n3 LCPUFAs from fish oil versus linseed or soybean oil was significantly more effective. Taken together, this evidence suggests that in addition to the dose of n-3 LCPUFAs, the proportion of EPA/DHA, ideally from marine sources, is also important.

Another question that remains is whether the baseline maternal LCPUFA levels were already too high pre-intervention. There was considerable variation in the pre-intervention levels of EPA, DHA, AA, and n-6/n-3 LCPUFAs across participants (16). Nonetheless, we did not control for pre-levels in analysis which could have obscured an intervention effect. A recent study by Vinding et al. (45) found that prenatal n-3 LCPUFA supplementation had an overall effect on offspring somatic growth at 6 years after controlling for baseline maternal n3 LCPUFA levels. However, it is interesting that they did not observe an interaction effect between maternal pre- and post intervention n-3 LCPUFA levels on child body composition (45).

There is also some debate as to whether women with high baseline n-3 LCPUFA levels should be excluded from intervention trials. A review that examined the efficacy of n-3 LCPUFA supplementation on reducing cardiac mortality concluded that there may be a threshold pre-intervention level in which n-3 LCPUFAs no longer have an effect (48). Given these findings, future LCPUFA intervention studies should consider routinely measuring baseline concentrations and statistically controlling for pre-intervention levels. Moreover, more research is needed to understand whether baseline n-3 LCPUFA concentrations modify relationships between n-3 LCPUFA supplementation and child outcomes $(10,49-51)$.

It is worthwhile to note that most studies began their intervention in the second trimester of pregnancy (38-40). Evidence of human adipocyte development has been observed 
between the 14th and 16th week of gestation (52), which supports the INFAT intervention start-time of $\sim 15$ weeks' gestation. However, maternal nutrition in the first trimester is critical for the growth and development of certain vital fetal organs (53). Early changes in maternal LCPUFA levels could have a programming effect on offspring, and some researchers have questioned whether an intervention should start earlier in pregnancy (54). First trimester interventions would entail recruiting women in the preconception period, which could be achieved by engaging primary care providers to actively participate in developing and implementing perinatal intervention trials.

\section{DIRECTION FOR FUTURE RESEARCH: TARGETING LCPUFA INTERVENTIONS FOR HIGH-RISK POPULATIONS}

Despite preclinical evidence demonstrating links between perinatal nutrition and an increased risk of offspring obesity and related diseases (13), research in humans has been less convincing. Most of the supporting evidence has been derived from epidemiological studies. Importantly, this relationship is often observed in circumstances of extreme under- or overnutrition, such as offspring of mothers who survived the Dutch Famine (1944-1945) $(55,56)$, which calls into question whether non-extreme alterations in perinatal nutrient availability are causally implicated in the development of chronic obesity-related diseases, or if other exposures in utero and early life are at play. Thus far, evidence that perinatal LCPUFA supplementation ameliorates offspring obesity outcomes in humans is largely unsubstantiated (38-40). However, it is unclear whether certain high-risk subgroups could benefit from $\mathrm{n}-3$ supplementation and insights gained from the INFAT study could help shape new directions for research.

There is strong evidence that supports prenatal n-3 LCPUFA supplementation to reduce the incidence of preterm birth (16, 57-60), especially in high-risk pregnancies $(60,61)$. A recent study of 184 countries reported that countries with low n3 LCPUFA dietary intake have a higher overall incidence of preterm births (62). Importantly, the researchers observed a threshold benefit to n-3 LCPUFAs, where no associations were observed between preterm birth incidence and countries whose dietary intake was above $600 \mathrm{mg} /$ day. Over $95 \%$ of American childbearing-aged women do not meet the current recommendation of $250 \mathrm{mg}$ n-3 LCPUFAs per day (63), with similar findings reported in Europe (64). Research has shown that a total percentage of $\leq 4.1 \%$ of $n-3$ fatty acids in maternal blood in early pregnancy strongly predicts preterm birth (65). These findings provide evidence that support n-3 LCPUFA interventions in populations whose daily consumption of $n$ 3 LCPUFAs falls short of the recommended intake, or for women with high-risk pregnancies $(61,65-67)$. Based on the aforementioned multi-country study by Ciesielski et al. (62), it is also worth considering revising the daily recommendation of n-3 LCPUFAs to $600 \mathrm{mg}$ during pregnancy to decrease the risk of preterm births, estimated to be $6.2 \%$ and $10.6 \%$ of all live births in Europe and North America, respectively $(62,68)$.

Another opportunity for targeted LCPUFA interventions is investigating outcomes in women with obesity. INFAT was designed as a concept validation study, therefore women with underweight or obesity were excluded. INFAT children were also leaner than their peers at 5 years, with only one child ( $\sim 1 \%$ ) having obesity, a marked contrast to the German pediatric population with obesity rates around $3.3-5.4 \%$ at this age (69). N-3 LCPUFAs are known to exhibit anti-inflammatory and antiobesogenic properties in animals (70), although data in humans do not consistently confirm these associations. One study that provided DHA supplements to pregnant women with obesity or gestational diabetes found no significant effects on offspring adiposity outcomes (71). More data are needed to determine whether supplementation in pregnant women in higher BMI categories may be a promising intervention to reduce offspring obesity risk.

\section{CONCLUSION}

Findings from the INFAT study demonstrate that perinatal administration of n-3 LCPUFAs has only transient effects in early childhood, arguing against this dietary intervention as a strategy for the primary prevention of childhood obesity. Instead, research aims should shift toward investigating whether certain vulnerable and high-risk subgroups could benefit from n-3 LCPUFA interventions. Insights gained from INFAT and other studies discussed herein point to promising results and implications for planning future intervention studies. Irrespective of our study results, long-term follow-up studies are necessary to understand how and to what extent perinatal nutrition and consecutive changes of regulatory hormonal systems influence health and disease risk in childhood and, ultimately, over the life course.

\section{DATA AVAILABILITY STATEMENT}

The original contributions presented in the study are included in the article/supplementary material, further inquiries can be directed to the corresponding author/s.

\section{ETHICS STATEMENT}

The studies involving human participants were reviewed and approved by Technical University of Munich Ethics Committee. The patients/participants provided their written informed consent to participate in this study.

\section{AUTHOR CONTRIBUTIONS}

$\mathrm{HH}$ designed the study and supervised the project. DM wrote the paper with input from all authors. $\mathrm{BB}, \mathrm{CB}$, and $\mathrm{DM}$ designed the table and figure. All authors discussed the findings and contributed to the manuscript revision. All authors contributed to the article and approved the submitted version. 


\section{FUNDING}

Else Kröner-Fresenius Foundation, Bad Homburg, Germany; International Unilever Foundation, Hamburg, Germany, provided institutional funding; EU-funded EARNEST (Early Nutrition Programming Project) consortium (FOOD-CT-2005007036), administratively facilitated by Frank Wiens, provided a study grant; German Ministry of Education and Research via the Competence Network on Obesity (Kompetenznetz Adipositas, 01GI0842), Germany, provided a study grant; Danone ResearchCenter for Specialized Nutrition, Friedrichsdorf, Germany, provided non-financial support.

\section{REFERENCES}

1. Barker DJ. The origins of the developmental origins theory. J Int Med. (2007) 261:412-7. doi: 10.1111/j.1365-2796.2007.01809.x

2. Gluckman PD, Hanson MA, Cooper C, Thornburg KL. Effect of in utero and early-life conditions on adult health and disease. N Engl J Med. (2008) 359:61-73. doi: 10.1056/NEJMra0708473

3. Plagemann A. Toward a unifying concept on perinatal programming: vegetative imprinting by environment-dependant biocybernetogenesis. In Plagemann A, editor. Perinatal Programming. State of the art. Berlin; Boston, MA: De Gruyter. (2012). p. 243-82.

4. Whitaker RC. Predicting preschooler obesity at birth: the role of maternal obesity in early pregnancy. Pediatrics. (2004) 114:e29-36. doi: 10.1542/peds.114.1.e29

5. Ludwig DS, Rouse HL, Currie J. Pregnancy weight gain and childhood body weight: a within-family comparison. PLoS Med. (2013) 10:e1001521. doi: 10.1371/journal.pmed.1001521

6. Mamun AA, Mannan M, Doi SA. Gestational weight gain in relation to offspring obesity over the life course: a systematic review and bias-adjusted meta-analysis. Obes Rev. (2014) 15:338-47. doi: 10.1111/obr.12132

7. Dalrymple KV, Martyni-Orenowicz J, Flynn AC, Poston L, O'Keeffe M. Can antenatal diet and lifestyle interventions influence childhood obesity? A systematic review. Mater Child Nutr. (2018) 14:e12628. doi: $10.1111 / \mathrm{mcn} .12628$

8. Kabaran S, Besler HT. Do fatty acids affect fetal programming? J Health Popul Nutr. (2015) 33:14. doi: 10.1186/s41043-015-0018-9

9. Muhlhausler BS, Ailhaud GP. Omega-6 polyunsaturated fatty acids and the early origins of obesity. Current Opin Endocrinol Diabetes Obes. (2013) 20:5661. doi: 10.1097/MED.0b013e32835c1ba7

10. Massiera F, Saint-Marc P, Seydoux J, Murata T, Kobayashi T, Narumiya $\mathrm{S}$, et al. Arachidonic acid and prostacyclin signaling promote adipose tissue development: a human health concern? J Lipid Res. (2003) 44:2719. doi: 10.1194/jlr.M200346-JLR200

11. Simopoulos AP. An increase in the omega-6/omega-3 fatty acid ratio increases the risk for obesity. Nutrients. (2016) 8:128. doi: 10.3390/nu8030128

12. Ailhaud G, Guesnet P. Fatty acid composition of fats is an early determinant of childhood obesity: a short review and an opinion. Obesity Rev. (2004) 5:21-6. doi: 10.1111/j.1467-789X.2004.00121.x

13. Muhlhausler BS, Miljkovic D, Fong L, Xian CJ, Duthoit E, Gibson RA. Maternal omega-3 supplementation increases fat mass in male and female rat offspring. Front Genet. (2011) 2:48. doi: 10.3389/fgene.2011.00048

14. Hauner H, Brunner S. Early fatty acid exposure and later obesity risk. Curr Opin Clin Nutr Metab Care. (2015) 18:1137. doi: 10.1097/MCO.0000000000000143

15. Hauner H, Vollhardt C, Schneider KT, Zimmermann A, Schuster T, AmannGassner U. The impact of nutritional fatty acids during pregnancy and lactation on early human adipose tissue development. Rationale and design of the INFAT study. Ann Nutr Metab. (2009) 54:97-103. doi: 10.1159/000209267

16. Hauner H, Much D, Vollhardt C, Brunner S, Schmid D, Sedlmeier E-M, et al. Effect of reducing the n-6:n-3 long-chain PUFA ratio during pregnancy and lactation on infant adipose tissue growth within the first year of life:

\section{ACKNOWLEDGMENTS}

We are grateful to the mothers and children who participated in the INFAT study. Figure courtesy of Christina Brei. The figure is modified from the original figure published in the Ph.D. thesis of Christina Brei (https://nbn-resolving.org/urn/resolver.pl?urn: nbn:de:bvb:91-diss-20171130-1360854-1-3). Our sincere thanks also extend to current and former colleagues who contributed to the study: Ulrike Amann-Gassner, Christiane Vollhardt, Daniela Much, Stefanie Brunner, Ellen Heimberg, Karina Pusch, Daniela Schmid, Eva-Maria Sedlmeier, Lynne Stecher, and several master students.

an open-label randomized controlled trial. Am J Clin Nutr. (2012) 95:38394. doi: 10.3945/ajcn.111.022590

17. Brei C, Stecher L, Much D, Karla MT, Amann-Gassner U, Shen J, et al. Reduction of the n-6: n-3 long-chain PUFA ratio during pregnancy and lactation on offspring body composition: follow-up results from a randomized controlled trial up to 5 y of age. Am J Clin Nutr. (2016) 103:147281. doi: 10.3945/ajcn.115.128520

18. Much D, Brunner S, Vollhardt C, Schmid D, Sedlmeier EM, Bruderl M, et al. Effect of dietary intervention to reduce the $n-6 / n-3$ fatty acid ratio on maternal and fetal fatty acid profile and its relation to offspring growth and body composition at 1 year of age. Eur J Clin Nutr. (2013) 67:2828. doi: 10.1038/ejcn.2013.2

19. Much D, Brunner S, Vollhardt C, Schmid D, Sedlmeier E-M, Brüderl M, et al. Breast milk fatty acid profile in relation to infant growth and body composition: results from the INFAT study. Pediatr Res. (2013) 74:2307. doi: 10.1038/pr.2013.82

20. Meyer DM, Brei C, Stecher L, Much D, Brunner S, Hauner H. Associations between long-chain PUFAs in maternal blood, cord blood, and breast milk and offspring body composition up to 5 years: follow-up from the INFAT study. Eur J Clin Nutr. (2019) 73:458-64. doi: 10.1038/s41430-018-0388-3

21. Brei C, Much D, Heimberg E, Schulte V, Brunner S, Stecher L, et al. Sonographic assessment of abdominal fat distribution during the first year of infancy. Pediatr Res. (2015) 78:342-50. doi: 10.1038/pr.2015.108

22. Brei C, Much D, Brunner S, Stecher L, Hauner H. Longitudinal sonographic assessment of abdominal fat distribution from 2 to 5 years of age. Pediatr Res. (2018) 84:677-83. doi: 10.1038/s41390-018-0147-0

23. Brei C, Stecher L, Meyer DM, Young V, Much D, Brunner S, et al. Impact of dietary macronutrient intake during early and late gestation on offspring body composition at birth, 1, 3, and 5 years of age. Nutrients. (2018) 10:579. doi: 10.3390/nu10050579

24. Brei C, Stecher L, Brunner S, Ensenauer R, Heinen F, Wagner PD, et al. Impact of the n-6:n-3 long-chain PUFA ratio during pregnancy and lactation on offspring neurodevelopment: 5-year follow-up of a randomized controlled trial. Eur J Clin Nutr. (2017) 71:1114-20. doi: 10.1038/ejcn.2017.79

25. Sedlmeier EM, Brunner S, Much D, Pagel P, Ulbrich SE, Meyer HH, et al. Human placental transcriptome shows sexually dimorphic gene expression and responsiveness to maternal dietary n-3 long-chain polyunsaturated fatty acid intervention during pregnancy. BMC Genomics. (2014) 15:941. doi: 10.1186/1471-2164-15-941

26. Brunner S, Schmid D, Hüttinger K, Much D, Heimberg E, Sedlmeier E$\mathrm{M}$, et al. Maternal insulin resistance, triglycerides and cord blood insulin in relation to post-natal weight trajectories and body composition in the offspring up to 2 years. Diabet Med. (2013) 30:1500-7. doi: 10.1111/dme.12298

27. Meyer DM, Brei C, Stecher L, Brunner S, Hauner H. Maternal insulin resistance, triglycerides and cord blood insulin are not determinants of offspring growth and adiposity up to 5 years: a follow-up study. Diabet Med. (2018) 35:1399-403. doi: 10.1111/dme.13765

28. Brunner S, Schmid D, Huttinger K, Much D, Bruderl M, Sedlmeier EM, et al. Effect of reducing the n-6/n-3 fatty acid ratio on the maternal and fetal leptin axis in relation to infant body composition. Obesity. (2014) 22:21724. doi: 10.1002/oby. 20481 
29. Meyer DM, Brei C, Stecher L, Much D, Brunner S, Hauner H. Leptin in maternal plasma and cord blood as a predictor of offspring adiposity at 5 years: a follow-up study. Obesity. (2018) 26:279-83. doi: 10.1002/oby.22037

30. Brunner S, Schmid D, Zang K, Much D, Knoeferl B, Kratzsch J, et al. Breast milk leptin and adiponectin in relation to infant body composition up to 2 years. Pediatr Obes. (2015) 10:67-73. doi: 10.1111/j.2047-6310.2014.222.x

31. Meyer DM, Brei C, Stecher L, Much D, Brunner S, Hauner H. The relationship between breast milk leptin and adiponectin with child body composition from 3 to 5 years: a follow-up study. Pediatr Obes. (2017) 12(Suppl 1):1259. doi: 10.1111/ijpo.12192

32. Meyer DM, Brei C, Stecher L, Much D, Brunner S, Hauner H. Cord blood and child plasma adiponectin levels in relation to childhood obesity risk and fat distribution up to 5 y. Pediatr Res. (2017) 81:745-51. doi: 10.1038/pr.2016.275

33. Rolland-Cachera MF, Deheeger M, Maillot M, Bellisle F. Early adiposity rebound: causes and consequences for obesity in children and adults. Int $J$ Obes. (2006) 30(Suppl 4):S11-7. doi: 10.1038/sj.ijo.0803514

34. Geserick M, Vogel M, Gausche R, Lipek T, Spielau U, Keller E, et al. Acceleration of BMI in early childhood and risk of sustained obesity. $N$ Engl J Med. (2018) 379:1303-12. doi: 10.1056/NEJMoa1803527

35. Dunstan JA, Simmer K, Dixon G, Prescott SL. Cognitive assessment of children at age $2(1 / 2)$ years after maternal fish oil supplementation in pregnancy: a randomised controlled trial. Arch Dis Child Fetal Neonatal Ed. (2008) 93:F45-50. doi: 10.1136/adc.2006.099085

36. Lauritzen L, Hoppe C, Straarup EM, Michaelsen KF. Maternal fish oil supplementation in lactation and growth during the first 2.5 years of life. Pediatr Res. (2005) 58:235-42. doi: 10.1203/01.PDR.0000169978.92437.58

37. Mook-Kanamori DO, Holzhauer S, Hollestein LM, Durmus B, Manniesing R, Koek M, et al. Abdominal fat in children measured by ultrasound and computed tomography. Ultrasound Med Biol. (2009) 35:1938-46. doi: 10.1016/j.ultrasmedbio.2009.07.002

38. Stratakis N, Gielen M, Chatzi L, Zeegers MP. Effect of maternal n-3 long-chain polyunsaturated fatty acid supplementation during pregnancy and/or lactation on adiposity in childhood: a systematic review and metaanalysis of randomized controlled trials. Eur J Clin Nutr. (2014) 68:127787. doi: $10.1038 /$ ejcn. 2014.158

39. Delgado-Noguera MF, Calvache JA, Bonfill Cosp X, Kotanidou EP, Galli-Tsinopoulou A. Supplementation with long chain polyunsaturated fatty acids (LCPUFA) to breastfeeding mothers for improving child growth and development. Cochrane Database Syst Rev. (2015) 7:Cd007901. doi: 10.1002/14651858.CD007901.pub3

40. Vahdaninia M, Mackenzie H, Dean T, Helps S. The effectiveness of $\omega$ 3 polyunsaturated fatty acid interventions during pregnancy on obesity measures in the offspring: an up-to-date systematic review and meta-analysis. Eur J Nutr. (2018) 58:2597-613. doi: 10.1007/s00394-018-1824-9

41. Grønn M, Gørbitz C, Christensen E, Levorsen A, Ose L, Hagve TA, et al. Dietary n-6 fatty acids inhibit the incorporation of dietary n-3 fatty acids in thrombocyte and serum phospholipids in humans: a controlled dietetic study. Scand J Clin Lab Invest. (1991) 51:255-63. doi: 10.3109/00365519109091612

42. Vries PS de, Gielen M, Rizopoulos D, Rump P, Godschalk R, Hornstra $\mathrm{G}$, et al. Association between polyunsaturated fatty acid concentrations in maternal plasma phospholipids during pregnancy and offspring adiposity at age 7: the MEFAB cohort. Prostaglandins Leukot Essent Fatty Acids. (2014) 91:81-5. doi: 10.1016/j.plefa.2014.04.002

43. Hense S, Pohlabeln H, Michels N, Mårild S, Lissner L, Kovacs E, et al. Determinants of attrition to follow-up in a multicentre cohort study in children-results from the IDEFICS study. Epidemiol Res Int. (2013) 2013:19. doi: $10.1155 / 2013 / 936365$

44. EFSA Panel on Dietetic Products Nutrition and Allergies. Scientific Opinion on Dietary Reference Values for fats, including saturated fatty acids, polyunsaturated fatty acids, monounsaturated fatty acids, trans fatty acids, and cholesterol. EFSA J. (2010) 8:1461. doi: 10.2903/j.efsa.2010.1461

45. Vinding RK, Stokholm J, Sevelsted A, Sejersen T, Chawes BL, Bønnelykke $\mathrm{K}$, et al. Effect of fish oil supplementation in pregnancy on bone, lean, and fat mass at six years: randomised clinical trial. BMJ. (2018) 362:k3312. doi: 10.1136/bmj.k3312

46. Dasilva G, Pazos M, García-Egido E, Gallardo JM, Rodríguez I, Cela $R$, et al. Healthy effect of different proportions of marine $\omega-3$ PUFAs EPA and DHA supplementation in Wistar rats: lipidomic biomarkers of oxidative stress and inflammation. J Nutr Biochem. (2015) 26:138592. doi: 10.1016/j.jnutbio.2015.07.007

47. Molinar-Toribio E, Pérez-Jiménez J, Ramos-Romero S, Romeu M, Giralt M, Taltavull N, et al. Effect of n-3 PUFA supplementation at different EPA:DHA ratios on the spontaneously hypertensive obese rat model of the metabolic syndrome. Br J Nutr. (2015) 113:878-87. doi: 10.1017/S0007114514004437

48. Meyer BJ, Groot RH de. Effects of omega-3 long chain polyunsaturated fatty acid supplementation on cardiovascular mortality: the importance of the dose of DHA. Nutrients. (2017) 9:1305. doi: 10.3390/nu9121305

49. Schebb NH, Ostermann AI, Yang J, Hammock BD, Hahn A, Schuchardt JP. Comparison of the effects of long-chain omega-3 fatty acid supplementation on plasma levels of free and esterified oxylipins. Prostaglandins Other Lipid Mediat. (2014) 113-115:21-9. doi: 10.1016/j.prostaglandins.2014.05.002

50. Ghandour RA, Colson C, Giroud M, Maurer S, Rekima S, Ailhaud G, et al. Impact of dietary $\omega 3$ polyunsaturated fatty acid supplementation on brown and brite adipocyte function. J Lipid Res. (2018) 59:45261. doi: 10.1194/jlr.M081091

51. Ostermann AI, Schebb NH. Effects of omega-3 fatty acid supplementation on the pattern of oxylipins: a short review about the modulation of hydroxy-, dihydroxy-, and epoxy-fatty acids. Food Funct. (2017) 8:235567. doi: 10.1039/C7FO00403F

52. Poissonnet CM, Burdi AR, Bookstein FL. Growth and development of human adipose tissue during early gestation. Early Hum Dev. (1983) 8:111. doi: 10.1016/0378-3782(83)90028-2

53. Institute of Medicine. Nutrition during Pregnancy. Washington, DC: National Academy Press (1990).

54. Blumfield ML. Can long-chain PUFA supplementation during pregnancy influence later obesity risk? Am J Clin Nutr. (2016) 103:1387-8. doi: 10.3945/ajen.116.136093

55. Painter RC, Roseboom TJ, Bleker OP. Prenatal exposure to the Dutch famine and disease in later life: an overview. Reprod toxicol. (2005) 20:34552. doi: 10.1016/j.reprotox.2005.04.005

56. Schulz LC. The Dutch Hunger Winter and the developmental origins of health and disease. Proc Natl Acad Sci U S A. (2010) 107:167578. doi: 10.1073/pnas.1012911107

57. Arntzen KJ, Brekke OL, Vatten L, Austgulen R. Reduced production of PGE2 and PGF2 alpha from decidual cell cultures supplemented with N3 polyunsaturated fatty acids. Prostaglandins Other Lipid Mediat. (1998) 56:183-95. doi: 10.1016/S0090-6980(98)00048-3

58. Kar S, Wong M, Rogozinska E, Thangaratinam S. Effects of omega-3 fatty acids in prevention of early preterm delivery: a systematic review and metaanalysis of randomized studies. Eur J Obstet Gynecol Reprod Biol. (2016) 198:40-6. doi: 10.1016/j.ejogrb.2015.11.033

59. Saccone G, Saccone I, Berghella V. Omega-3 long-chain polyunsaturated fatty acids and fish oil supplementation during pregnancy: which evidence? J Matern Fetal Neonatal Med. (2016) 29:2389-97. doi: 10.3109/14767058.2015.1086742

60. Middleton P, Gomersall JC, Gould JF, Shepherd E, Olsen SF, Makrides M. Omega-3 fatty acid addition during pregnancy. Cochrane Database Syst Rev. (2018) 11:CD003402. doi: 10.1002/14651858.CD003402.pub3

61. Horvath A, Koletzko B, Szajewska H. Effect of supplementation of women in high-risk pregnancies with long-chain polyunsaturated fatty acids on pregnancy outcomes and growth measures at birth: a metaanalysis of randomized controlled trials. Br J Nutr. (2007) 98:2539. doi: 10.1017/S0007114507709078

62. Ciesielski TH, Bartlett J, Williams SM. Omega-3 polyunsaturated fatty acid intake norms and preterm birth rate: a cross-sectional analysis of 184 countries. BMJ Open. (2019) 9:e027249. doi: 10.1136/bmjopen-2018-027249

63. Zhang Z, Fulgoni VL, Kris-Etherton PM, Mitmesser SH. Dietary intakes of EPA and DHA omega-3 fatty acids among US childbearing-age and pregnant women: an analysis of NHANES 2001-2014. Nutrients. (2018) 10:416. doi: 10.3390/nu10040416

64. Sioen I, van Lieshout L, Eilander A, Fleith M, Lohner S, Szommer A, et al. Systematic review on N-3 and N-6 polyunsaturated fatty acid intake in european countries in light of the current recommendations - focus on specific population groups. Ann Nutr Metab. (2017) 70:39-50. doi: 10.1159/000456723

65. Simmonds LA, Sullivan TR, Skubisz M, Middleton PF, Best KP, Yelland $\mathrm{LN}$, et al. Omega-3 fatty acid supplementation in pregnancy-baseline 
omega-3 status and early preterm birth: exploratory analysis of a randomised controlled trial. BJOG Int J Obstet Gynaecol. (2020) 127:97581. doi: 10.1111/1471-0528.16168

66. Giuseppe $\mathrm{R}$ de, Roggi C, Cena H. n-3 LC-PUFA supplementation: effects on infant and maternal outcomes. Eur J Nutr. (2014) 53:114754. doi: 10.1007/s00394-014-0660-9

67. Huffman SL, Harika RK, Eilander A, Osendarp SJ. Essential fats: how do they affect growth and development of infants and young children in developing countries? A literature review. Matern Child Nutr. (2011) 7(Suppl 3):4465. doi: 10.1111/j.1740-8709.2011.00356.x

68. Beck S, Wojdyla D, Say L, Betran AP, Merialdi M, Requejo JH, et al. The worldwide incidence of preterm birth: a systematic review of maternal mortality and morbidity. Bull World Health Organ. (2010) 88:318. doi: 10.2471/BLT.08.062554

69. Moss A, Klenk J, Simon K, Thaiss H, Reinehr T, Wabitsch M. Declining prevalence rates for overweight and obesity in German children starting school. Eur J Pediatr. (2012) 171:289-99. doi: 10.1007/s00431-011-1531-5

70. Muhlhausler BS, Gibson RA, Makrides M. The effect of maternal omega-3 long-chain polyunsaturated fatty acid (n-3 LCPUFA) supplementation during pregnancy and/or lactation on body fat mass in the offspring: a systematic review of animal studies. Prostaglandins Leukotrienes Essential Fatty Acids. (2011) 85:83-8. doi: 10.1016/j.plefa.2011.04.027

71. Foster BA, Escaname E, Powell TL, Larsen B, Siddiqui SK, Menchaca J, et al. Randomized controlled trial of DHA supplementation during pregnancy: child adiposity outcomes. Nutrients. (2017) 9:566. doi: 10.3390/nu90 60566

Conflict of Interest: The authors declare that the research was conducted in the absence of any commercial or financial relationships that could be construed as a potential conflict of interest.

Copyright (C) 2020 Meyer, Brei, Bader and Hauner. This is an open-access article distributed under the terms of the Creative Commons Attribution License (CC BY). The use, distribution or reproduction in other forums is permitted, provided the original author(s) and the copyright owner(s) are credited and that the original publication in this journal is cited, in accordance with accepted academic practice. No use, distribution or reproduction is permitted which does not comply with these terms. 